\title{
WiMAX in the Classroom: Designing a Cellular Networking Hands-on Lab
}

\author{
Jelena Marašević*, Jan Janak ${ }^{\dagger}$, Henning Schulzrinne ${ }^{\dagger}$, Gil Zussman* \\ ${ }^{*}$ Department of Electrical Engineering, ${ }^{\dagger}$ Department of Computer Science \\ Columbia University, New York, NY \\ \{jelena, gil\}@ee.columbia.edu, \{janakj, hgs $\} @$ cs.columbia.edu
}

\begin{abstract}
Wireless networking has recently gained tremendous attention in research and education. Since the concepts taught in wireless courses are difficult to acquire only through lectures, hands-on lab experience is indispensable. While Wi-Fi based networking labs have been introduced before, to the best of our knowledge, labs that use a cellular technology have not been designed yet. Therefore, we present a WiMAX hands-on lab designed for a graduate course in wireless and mobile networking. The lab is based on the mobile WiMAX hardware and software developed and deployed within the GENI WiMAX project. We provide a brief overview of the course and of the main concepts taught in the WiMAX lecture. Then, we describe in detail our WiMAX network and the structure of the lab experiment. The effectiveness in achieving the learning objectives is evaluated via the lab reports submitted by the students. Finally, we review some of the lessons we learned during design and implementation of this lab. These can provide important insights to designers of similar labs.
\end{abstract}

Index Terms-Teaching, hands-on lab, networking, WiMAX, cellular.

\section{INTRODUCTION}

Wireless and mobile technology has been progressing rapidly in recent years. A large number of applications, along with increased interest in industry and academia, have made wireless networking an attractive and vibrant area.

Courses on wireless and mobile networking have been gaining popularity. The Electrical Engineering Department at Columbia University offers a graduate level course "ELEN E6951: Wireless \& Mobile Networking II". The students learn selected topics in wireless and mobile networks through a mix of lectures, project work, and hands-on lab assignments.

The importance of lab experiments in teaching networking courses has been widely recognized, and labs have become an integral part of such courses [1]-[7]. Evaluations presented in [2]-[7] suggest that the lab experience makes the learning process easier and more exciting, and students obtain better understanding of networking concepts. Most labs designed for wireless networking courses focus entirely on Wi-Fi. We have also designed Wi-Fi labs for our course in the past.

Considering almost universal ubiquity of mobile networks, we decided to extend the course with another hands-on lab. Our aim is to expose the students to the technology used in 4G wireless networks. We chose WiMAX as the representative technology, mainly since we have access to a dedicated
WiMAX base station on our campus. ${ }^{1}$ The learning objective is to highlight some of the differences between WiMAX and Wi-Fi through an experiment involving a real mobile WiMAX Base Station (BS) and two Mobile Station (MS) nodes. To the best of our knowledge, this is the first networking hands-on lab that uses a cellular technology.

In this paper, we present the initial design and evaluation of a WiMAX hands-on lab based on an open, programmable WiMAX BS developed by the GENI WiMAX project [8]. Moreover, we review the lessons learned as possible guidelines for other instructors designing similar courses.

The open nature of the BS lets students experiment with various parameters in real-time and observe their influence on the ongoing experiment. Most mobile WiMAX deployments are operated by commercial providers (e.g., Clearwire [9]). Therefore, it is impossible for students to get a glimpse into the operation of the BS, as commercial providers would hardly grant this level of access. Hence, we believe that we provide the students with a valuable experience, as they interact with the real network equipment, observing the real data. Furthermore, our hands-on lab is not limited to on-campus students. It is also offered remotely through Columbia Video Network $(\mathrm{CVN})$. Providing hands-on experience to remote students will become increasingly important as distant learning gains more popularity.

The rest of the paper is organized as follows. We briefly review related work in Section II. In Section III we present the overall structure of the course. Section IV provides an overview of the WiMAX testbed used in the hands-on lab. Section $\mathrm{V}$ provides a detailed overview of the hands-on lab. We evaluate the design and implementation of the hands-on lab in Section VI, describe the lessons learned in Section VII, and conclude in Section VIII. We provide the lab materials, including pre-lab questions and lab instructions, in appendices I and II, respectively.

\section{RELATED WORK}

Most of the networking lab experiments focus either on Internet protocols [1]-[5], or on the Media Access Control (MAC) and physical layer (PHY) mechanisms in Wi-Fi [6], [7]. The work on designing labs that teach cellular networking

\footnotetext{
${ }^{1}$ Nevertheless, Long Term Evolution (LTE), the other competing standard, is based on similar concepts.
} 
concepts is rather sparse. Software tools such as OPNET [10] have capabilities for modeling and simulation of different cellular standards. However, to the best of our knowledge, all the existing cellular networking labs focus on the physical layer and cellular network planning (e.g., [11], [12]).

Recently, [13] proposed a high-level design of a WiMAX lab for networking courses. Even though the lab is envisioned to cover the networking aspects, no actual implementation is presented in [13].

To the best of our knowledge, networking aspects, such as MAC layer operation, throughput, and Quality of Service (QoS) provisioning in cellular networks, have not been covered by labs in networking courses before.

\section{Course Structure}

This section provides a high-level overview of the wireless and mobile networking course. We describe students' background prior to the lab, and place cellular technology in the context of the course. We describe students' background prior to the lab, and place cellular technology in the context of the course.

Wireless \& Mobile Networking II is a research-oriented graduate level course that covers various topics, mostly focusing on functionalities of the layers above the physical layer. The objective is to provide students with an understanding of the latest wireless network design challenges, protocols, and proposed algorithms. The topics include:

- Wireless communications basics (brief review);

- MAC protocols (e.g., TDMA, Aloha, CSMA/CA);

- Recent standards (e.g., IEEE 802.11, Bluetooth/IEEE 802.15, IEEE 802.16);

- Wireless networking concepts (ad hoc networks, wireless mesh networks, sensor networks, vehicular networks, cognitive radio networks, etc.);

- Routing protocols;

- Transport protocols (TCP over wireless, flow and congestion control in wireless);

- Energy Management;

- Localization;

- Time synchronization;

- Cross layer design (joint routing, scheduling, channel allocation, etc.);

- Fundamental limitations (capacity, connectivity, etc.);

- Mobility models and mobility control.

Typically, about 20 graduate students attend the class on campus, and several additional students take the course remotely through the Columbia Video Network (CVN). Most students have electrical engineering or computer science background. On-campus students are in graduate, mostly M.Sc., programs. CVN students are typically working professionals.

During the semester, students complete 3 problem sets, 3 hands-on labs, and one programming (simulation) assignment. The students are also required to work on a project.

In the simulation assignment, the students develop a simulation of an Aloha-based MAC protocol, and evaluate the performance (throughput, collision probability) in different

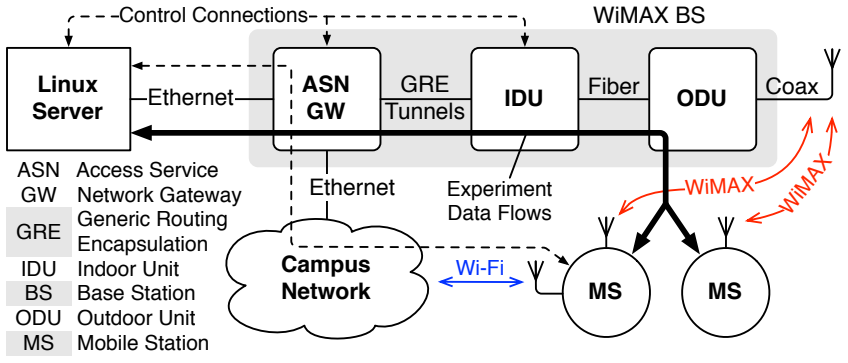

Fig. 1. WiMAX Base Station (BS) and network architecture used for the hands-on lab. Experiment data flows between the Linux Server and Mobile Stations (MS) via the BS. One MS is connected to the campus Wi-Fi network and maintains a control connection to the Linux Server. The connection is used to control experiments and monitor the BS.

scenarios. This provides them with an intuition on randomaccess MAC protocols, and allows them to confirm the results they obtain analytically in the problem sets.

There are two Wi-Fi and one WiMAX hands-on labs. Each lab is preceded by a pre-lab assignment, whose main objective is to ensure that the students are familiar with the Linux commands used in the lab, and that they have a basic understanding of the material covered. Pre-lab is assigned one week before the corresponding lab starts, and students are given one week to complete it. Every student is assigned a 3-hour time slot and provided with detailed written instructions, formulated in a step-by-step manner. The students are asked to collect relevant data and answer questions about the results obtained during the experiments. Upon completion of the hands-on lab, students are required to submit a lab report. The lab report is due 1-2 weeks after the lab is completed, providing students with enough time to reason about the data collected in the lab. The lab reports allow us to evaluate the work, and also provide feedback on the instructiveness of the lab assignment, the students' ability to understand and properly interpret the results, and their overall understanding of the learned concepts.

\section{WiMAX NETWORK CONFIGURATION}

We designed a simple WiMAX network to be used in our hands-on lab. The network consists of the following components:

- Two laptops used as Mobile Station (MS) nodes.

- A dedicated mobile WiMAX Base Station (BS).

- A Linux server used as a BS monitor and traffic sink or generator.

Fig. 1 illustrates the overall network architecture, as well as the main components of the BS.

The BS is an open, programmable mobile WiMAX base station developed within the GENI WiMAX project [8]. It has been designed for research and educational purposes, and is deployed by a number of universities across the U.S. The hardware and selected software components are based on a mobile WiMAX BS developed by NEC. The BS is composed of: the Outdoor Unit (ODU), the Indoor Unit (IDU), and the Access Service Network Gateway (ASN GW). Functions 


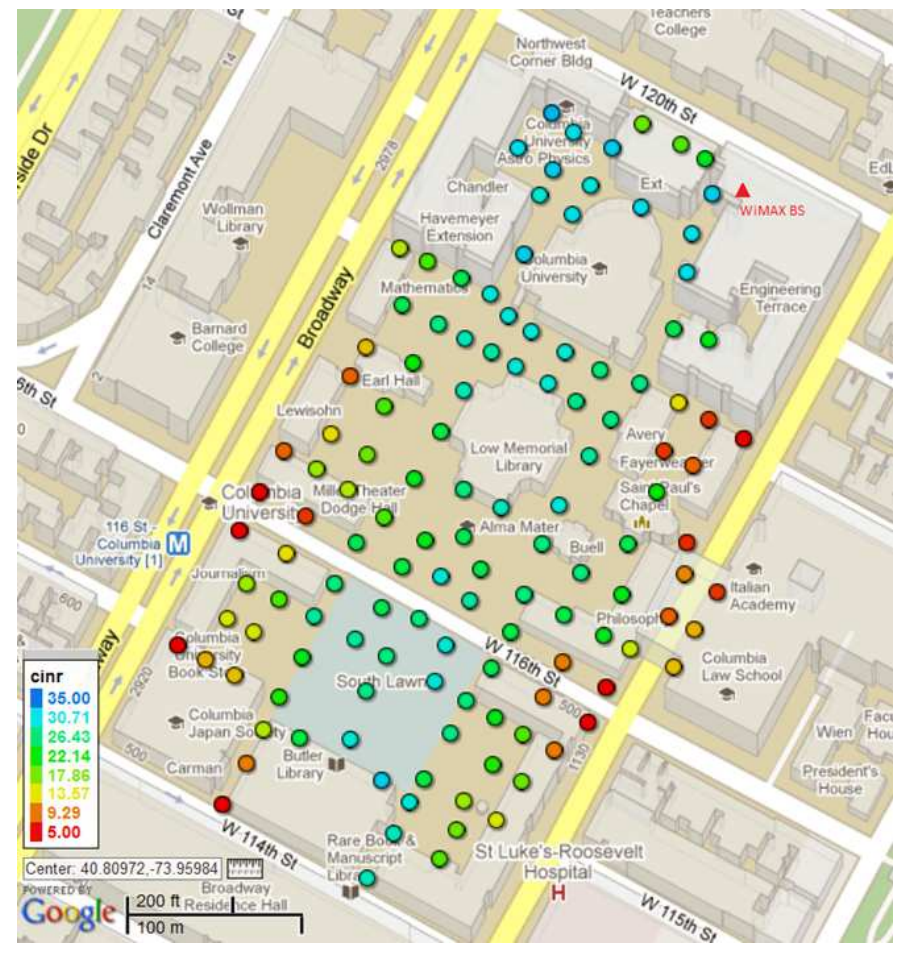

Fig. 2. Downlink Carrier to Interference-plus-Noise Ratio (CINR) coverage map of Columbia University campus. Blue and green dots indicate good signal reception. Yellow and red dots indicate weak or distorted signal. One of the Mobile Stations used for the hands-on lab was used to measure CINR.

and interfaces of those components are in accordance with the WiMAX reference network architecture [14]. Each of the components runs on a dedicated computer. Certain parts of the BS, such as the ASN GW, have been re-implemented by the GENI WiMAX team using open software, namely Ubuntu Linux and Click modular router [15].

The BS is installed on the $16^{\text {th }}$ floor of Mudd building at the Columbia University campus [16]. It operates in the $2.5 \mathrm{GHz}$ band under an experimental license with a $120^{\circ}$ sector antenna. The output power is set to $30 \mathrm{~W}$, which proved to be sufficient to cover the entire campus, including many indoor areas. Under optimal signal conditions the BS provides a downlink bandwidth of $12 \mathrm{Mbps}$ and an uplink bandwidth of about $1.5 \mathrm{Mbps}$ to the MSs. The uplink-downlink bandwidth ratio is configurable. The downlink Carrier to Interferenceplus-Noise Ratio (CINR) coverage map is shown in Fig. 2. For the purposes of the lab, we selected two locations on the campus, one with consistently strong WiMAX signal, and the other with weak (but usable) signal levels.

The entire BS operates at layer 2 (below the IP layer). For each associated MS, the IDU creates a Generic Routing Encapsulation (GRE) tunnel from the ASN GW to the MS. Thus, each MS, once associated, has a virtual network interface on the ASN GW node, as shown in Fig. 1. IP packet forwarding between the MS interface and other interfaces (Campus Network and Linux Server) is realized with the Click modular router. The BS is directly connected to the campus network, without any additional network address translators
TABLE I

Mobile Station System Configuration

\begin{tabular}{|c|c|c|}
\hline & MS 1 & MS 2 \\
\hline Hardware & Dell Laptop & Asus Eee PC \\
\hline OS & Ubuntu 11.04 & Ubuntu 10.10 \\
\hline WiMAX NIC & Intel Centrino WiMAX & Intel Centrino WiMAX \\
& 6250 & 6250 \\
\hline WiMAX Driver & Intel wimaxcu v1.5.1 & Intel wimaxcu v1.5.1 \\
\hline Testing Tool & Iperf 2.0.5 & Iperf 2.0.4 \\
\hline
\end{tabular}

or application level gateways. MSs connected to the BS can obtain a public IP address via Dynamic Host Configuration Protocol (DHCP) and appear as being directly connected to the campus network by an Ethernet switch.

Network configuration on both MSs is intentionally left to the students. We use a private IP address space to isolate the WiMAX network from the campus network. Even though the system supports DHCP with public addresses assigned by the campus DHCP server, we instruct students to configure the WiMAX network name, IP address and related parameters on both MSs manually each time they run an experiment. This is to ensure that all the experiments are repeatable.

The Linux server, attached directly to the BS via a dedicated Gigabit Ethernet interface, provides the interface for the students to interact with the BS. We provide Secure Shell (SSH) access to the server. Students login to the server from a MS via its Wi-Fi interface (connected to campus network), bypassing the often reconfigured WiMAX interface. Experiment data flows are generated with Iperf installed on the server. The server is used both as a traffic generator (for downlink experiments) and as a traffic sink (for uplink experiments). A dedicated Ethernet connection to the BS prevents any influence of traffic coming from the campus network on the experiments.

Collecting run-time data from the BS requires logging into both the ASN GW and the IDU, as well as running multiple commands on both. Therefore, we provide convenience scripts for students to hide the lower-level mechanics of obtaining data from the BS. We prepared two scripts (described in Section V-C) on the server to monitor the status of the BS and to obtain information about the WiMAX channel. Students use those scripts to observe the WiMAX channel utilization during experiments, and to monitor various channel parameters adjusted by the BS in response to varying signal conditions.

We use two laptops equipped with Intel WiMAX miniPCI cards as MSs (see Table I for configuration details). Both laptops have a built-in WiMAX antenna and are running Ubuntu Linux in the default configuration with the Intel WiMAX drivers compiled from the source code. The WiMAX Network Interface Card (NIC) presents itself as a dedicated Ethernet controller to the operating system. WiMAX related parameters can be configured by a command line tool.

To expose the students to the effects of various Quality of Service (QoS) classes in WiMAX, we statically configured the BS to place each of the two MSs into a different traffic class. One of the MSs is configured to use the Unsolicited Grant Service (UGS) class for all its traffic. The other MS is always 
using the Best Effort (BE) service class. The students do not know in advance which MS belongs to which class. Their goal is to identify the MSs assignment to classes based on the data collected during the lab.

\section{WIMAX HANDS-ON LAB}

In this section we present the WiMAX components of the course: the WiMAX lecture, the pre-lab assignment, the hands-on lab design and experiments, and the lab report requirements. The hands-on lab was organized for the first time in the Spring semester 2012, and repeated having only CVN students in Summer 2012.

\section{A. Lecture}

A lecture is given to familiarize the students with WiMAX and to contrast it with $\mathrm{Wi}-\mathrm{Fi}$ and other wireless network technologies. In the lecture, we present a brief overview of the standard, explain the basic mechanisms used in the physical and MAC layers, and discuss the network architecture.

More specifically, we discuss Adaptive Modulation and Coding (AMC) and its influence on throughput achieved by mobile users. We introduce Multiple-Input-Multiple-Output (MIMO) as a new technology at the physical layer. We explain the main principles in Orthogonal Frequency Division Multiple Access (OFDMA), adopted by both LTE and WiMAX as a multiple-access scheme, and compare it to the random access scheme used in Wi-Fi. Finally, we highlight the differences between cellular systems, where the BS allocates resources to users, and contention-based systems, where all the users compete for a shared wireless channel. One of the main points in the experiment is the Quality of Service (QoS) provisioning in WiMAX. Therefore, we also provide students with an overview of different traffic classes, discuss their main characteristics and the types of traffic that are typically assigned a particular class.

In addition to the lecture, students are referred to the literature [14], [17] and online resources [18], [19].

\section{B. Pre-lab}

Students are assigned a pre-lab homework to ensure that they are well prepared for the lab and able to properly interpret results. Our experience with other labs has shown that a set of simple questions helps students focus on the relevant material while preparing for the lab. It also helps them recognize the key aspects of the experiments. These questions are intended to be basic, and students typically make very few or no mistakes.

The pre-lab questions are grouped into four parts:

1. Linux Commands. We ask students about Linux commands needed for the lab. For example, about the difference between "normal" ping and ping flooding, the meaning of individual values in the information about round trip time (rtt min/avg/max/mdev), and the parameters used to adjust packet size.

2. PHY Layer Parameters. During the lab, students obtain the values of Carrier to Interference-plus-Noise Ratio (CINR), Received Signal Strength Indication (RSSI), and information about the modulation scheme. Therefore, we ask about CINR and RSSI, their influence on the selected modulation scheme, and their effect on the throughput.

3. MAC Layer. In this part, we ask students to describe the differences between the Wi-Fi and WiMAX MAC. For example, we ask whether WiMAX MAC can guarantee a minimum bandwidth for a MS regardless of other traffic on the channel. Moreover, students are asked to explain which approach is better for a large number of users. There are also questions about the differences between Unsolicited Grant Service (UGS) and Best Effort (BE) QoS classes. In addition, students should determine the class that is preferred for Voice-Over-IP (VoIP) traffic.

4. Network Architecture. Students are presented with a diagram similar to Fig. 1 and referred to [14]. We ask them to deduce which box implements authorization, mobility management and QoS in a WiMAX network, and to place the label R6 (according to the WiMAX reference model) on one of the links.

The pre-lab assignment used for the class taught in Spring and Summer 2012 appear in the Appendix I.

\section{Instructions and Experiment Design Details}

On-campus students work in pairs. CVN (remote) students complete the lab by connecting to the laptops and the server via SSH. Students are provided with very detailed step-bystep instructions, that also include questions they are required to answer in the lab report.

As mentioned in Section IV, there are two MSs, and the lab is done at two locations-one with a weak signal, and one with a strong signal. Both locations were chosen to be indoors, due to practical reasons further discussed in Section VII. CVN students perform only a part of the experiment with statically installed laptops at the strong signal location.

The hands-on lab consists of three logical parts: system setup, maximum throughput measurement, and quality of service measurement. The instructions we used for the class appear in the Appendix II.

1) System Setup: The students manually configure the WiMAX settings at the beginning of the experiment at each location. More specifically, they complete the following steps: verify the status of WiMAX cards and the wireless link, scan for available networks, and connect to the network.

The next step is to bring WiMAX interfaces up, configure IP addresses for both laptops, and write down their MAC addresses. The MAC address is needed later to interpret status information provided by the BS. Students start Iperf in server mode on both laptops by running the command:

iperf $-i \quad 1-u-s$

and open the control SSH connection to the Linux Server from one of the laptops.

There are two scripts run on the server to monitor the state of the BS: wi_monitor and wi_throughput. The first script returns the information about the wireless channel, including RSSI, CINR and modulation for both uplink and downlink, while the second one shows the throughput details 


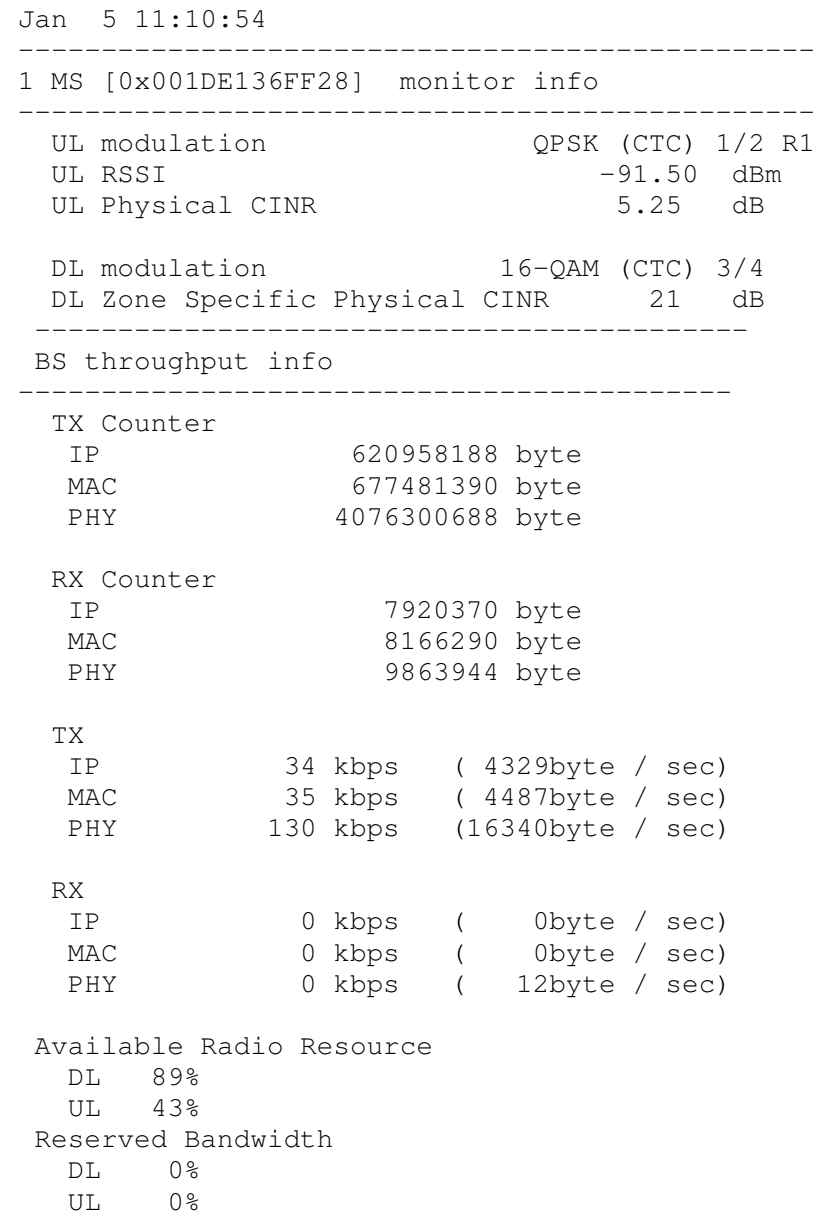

Fig. 3. Sample output of the wi_monitor and wi_throughput scripts.

of the wireless channel. The sample output obtained by running these scripts is shown in Fig. 3.

To complete the setup, students verify connectivity to the BS from both MSs with ping and subsequently with ping flooding using different packet sizes. For each test they note the average round trip time, as well as the sent and received packet statistics on both laptops.

2) Maximum Throughput Measurement: The objective of this experiment is to observe the difference in maximum throughput obtained by a single MS at the two locations. The intention is to show the influence of PHY parameters on the modulation scheme selected by the BS and its effect on the maximum throughput under a particular channel condition.

To observe the modulation scheme selected by the BS for a particular MS, students run the wi_monitor script. To obtain the information about the link status, such as CINR, RSSI, and average transmission power values, students run the wimaxcu status link command ${ }^{2}$ on the MS (laptop).

The Iperf testing tool is used to generate UDP data streams from the BS toward a MS and observe the throughput and packet loss. Students start Iperf in client mode on the Linux

\footnotetext{
${ }^{2}$ The command wimaxcu is part of the WiMAX Linux configuration tools provided by Intel.
}

Server to generate traffic towards the MS with the initial rate of 4 Mbps. They increase the rate in 1 Mbps steps and observe the packet loss. Based on the observation, they are asked to determine the maximum throughput for which the packet loss remains consistently below $2 \%$.

3) Quality of Service Measurement: The goal of this experiment is to identify which laptop was configured to use the Unsolicited Grant Service (UGS) service class. The instructions indicate that each laptop is using a different service class, without revealing the exact MS-QoS class assignment. This experiment is performed at the strong signal location only.

The BS uses different channel allocation policies for UGS and Best Effort (BE) clients. Students run two ping-based tests on both laptops and record the various RTT related values reported by ping. As the first step, students run a ping test on each laptop separately, to ensure that there is no other traffic on the channel during the test. The second step is to run a ping flooding test with a slightly larger packet size on both laptops at the same time. The goal of the second test is to emulate a WiMAX channel under load.

The UGS laptop normally reports a significantly shorter average RTT during the first test than the BE laptop. During the second test, the UGS laptop reports generally lower deviance from the average RTT. Students are asked to reason about the RTT numbers obtained on both laptops and, based on them, determine which laptop is using the UGS class.

\section{Lab Report}

All the data collected during the experiments is submitted in the form of a lab report. The report should also contain the answers to the following questions:

1. How does the delay you observed when you were pinging the BS compare to the delay you could see when you used Wi-Fi cards (in previous hands-on labs) instead of WiMAX? Can you explain the difference? When you execute ping flooding, do you observe different values of packet loss percentage for the two scenarios? Why is this the case?

2. What is the difference in the link quality at the two locations? What determines the attainable throughput at different locations? In what case do you get better performance and why?

3. Could you determine which machine was set to work with real-time traffic and which one was set to work as besteffort? What differences in ping delay did you observe? Why are these values different?

Lab reports help us verify that students understand the key aspects of the experiment, and also allow us to assess the success in achieving the learning objectives of the lab.

\section{Evaluations}

Based on the lab reports submitted by the students, we assessed their understanding of the material, and identified some issues related to the lab.

When asked about the difference in the average ping delay for Wi-Fi and WiMAX, some students wrote that WiMAX 
clients, on average, experience a lower ping delay, even though the average ping delay observed for WiMAX is several times higher. Some of the explanations they provided were that WiMAX is centralized, provides better QoS, has adaptive modulation and coding, etc. Not many students considered resource allocation, in which these two technologies differ. In particular, longer average ping delay is inherent in cellular technologies due to centralized allocation of resources, in which more control information needs to be exchanged, and transmission is allowed only within allocated time slots. The percentage of the students who answered this question incorrectly suggests that we should provide more background on the centralized control in the lecture. On the experiment side, we should make the differences in the average ping delay more apparent.

Most students understood well the differences in delay, throughput, and packet loss caused by different channel conditions at the two locations. At the same time, students had no difficulties with observing longer ping delays for larger packets, nor with explaining the influence of packet size on the ping delay.

We were very pleased to have all the students correctly determine which laptop was configured for the UGS traffic class, as well as provide a proper explanation of their conclusion. The answers provided by the students suggest that they acquired a good understanding of the differences between QoS classes and mechanisms of the WiMAX QoS provisioning.

From the data collected by students, we learned that controlling the CINR and the modulation scheme at MSs is very difficult under the current experimental setup. We aimed to use 16-QAM and QPSK as downlink and uplink modulations, respectively, at the first location, for both laptops. However, in some cases one laptop selected these modulations, while the other one selected QPSK for both uplink and downlink. This may pose challenges if the lab is extended in the future with a MSs performance comparison under a poor channel condition.

In the current setup this issue is insignificant. Namely, in the Maximum Throughput Measurement experiment (Section V-C2), conducted at both locations, only one MS is used as a client. On the other hand, the performance of MSs is compared based on the Quality of Service Measurement experiment (Section V-C3), which is conducted only at the second, strong signal, location. The second location allows 64-QAM and 16-QAM to be selected as modulations for downlink and uplink channels. Students are instructed to move the laptops around until the same modulations are selected for both laptops, and they reported no issues in achieving it.

During the lab design phase, we ran the experiments repeatedly to test all the commands and verify that obtained results conform to the behavior we would like to observe. Overall, the data collected by students agrees with the data we collected in the testing phase.

In general, we observe no differences in the data collected by the on-campus and CVN students for the part of the lab that they both complete. Except for performing experiments at only one location, CVN students do not seem to have any disadvantage when compared to the on-campus students, and they show no apparent difference in the knowledge acquired through the lab.

\section{LESSONS LEARNED}

Given that the lab was not built upon other teaching labs, we expected some challenges while preparing the network and during labs themselves. In this section we present some of the lessons learned in the hope that it will be useful for the designers of similar courses at other educational institutions.

One of our original ideas was to require the students to analyze layer 2 WiMAX frames and observe the messages being exchanged between the BS and the MS during the association process. Unfortunately, we were unable to do that because the WiMAX NIC does not provide access to the lowlevel WiMAX traffic. We evaluated four different types of WiMAX adapters for the lab, including one built-in into a smart phone. However, none of them provided the desired level of detail. We decided to use the Intel Centrino WiMAX 6250 NIC. The NIC emulates an Ethernet interface to the operating system, and all low-level operation of the card is driven by binary-only firmware loaded into the card at run-time. As of Spring 2012, Intel provided no interface to the low-level functionality of the card. To the best of our knowledge, such interface has not been added in the meantime.

Moreover, we were planning to use true mobile nodes, such as the Google Nexus $4 \mathrm{G}$ phone. Unfortunately, it was impossible to configure those phones to work with our GENI WiMAX base station, due to limitations in the phone software.

Another practical challenge was related to observing the behavior of UGS clients with reserved bandwidth under the channel overload (Section V-C3, Quality of Service Measurement). The idea was to let students see that the behavior of the UGS client is not affected by the traffic generated by other clients on the same channel. Although we configured the BS according to instructions, the bandwidth reservation feature was not entirely reliable. While the UGS client received preferential treatment compared to $\mathrm{BE}$ clients, the portion of the bandwidth reserved for the client varied. We were not able to investigate this behavior more, due to lack of documentation for that particular part of the system. We had to alter the experiment to accommodate for this issue, letting students focus on round-trip times, rather than on the available bandwidth under load.

Having a stable Wi-Fi connection at both locations was also challenging. In the location we picked for the weak signal experiment, the Wi-Fi connection to the server was sometimes flaky, and students had to reconnect repeatedly.

Selecting two locations, one with strong, and another one with weak, but still usable, signal level was not entirely trivial. Since the MSs were realized on laptops that students had to carry around, we wanted to avoid exposing the students to outdoor weather conditions. We also had to consider the availability of wall sockets at the two locations in case they run out of battery power. We had to select a different single location for the CVN students conducting the lab. We could 
not use the same locations as for the on-campus students, because this would require monitoring and moving the laptops by someone on campus. In the future, we may need to consider designing a more-controlled environment, possibly involving statically installed MSs that the students could access remotely via SSH. This would also enable the same treatment for the on-campus and CVN students.

Finally, we experienced a small number of connectivity issues with the WiMAX BS during one of the experiments. A restart of the BS always helped, but this required students to contact the TA for the lab, losing valuable time.

\section{CONCLUSIONS AND FUtURE WORK}

This paper describes the first in-class WiMAX hands-on lab designed for a course in wireless and mobile networking.

All student teams completed the experiments successfully. From the answers provided in the lab reports we could verify their level of understanding of the WiMAX technology, as well as obtain feedback on the design of the hands-on lab as a whole. Our lab is suitable for being conducted remotely, and has been offered to students via Columbia Video Network.

Nevertheless, there is considerable space for improvement in the design of the lab. Due to various limitations of our BS and NICs (described in detail in Section VII) we could not realize all the experiments we had originally envisioned. For the next iteration of the lab we would like to obtain better WiMAX cards for the MSs, or even consider using WiMAX equipped smart phones, such as the HTC Evo $4 \mathrm{G}$ available soon from the GENI WiMAX project. As mentioned before, we plan to explore the possibility of using permanently installed MSs that can be accessed remotely, with artificial signal attenuation on the MS side to simulate varying channel conditions. Based on such a design, one can consider deploying MSs in institutions with a WiMAX BS and allowing students from other universities to conduct the lab remotely.

On the server side, we will aim to obtain more documentation about some of the low-level features of the GENI WiMAX base station, in particular, documentation related to the QoS settings. This would enable us to extend our lab with more detailed experiments. For example, we plan to add comparisons of different QoS classes based on throughput, packet loss and delay under the real load.

We also plan to coordinate our WiMAX teaching efforts with other GENI WiMAX sites that are designing a similar hands-on lab.

Finally, the GENI WiMAX group has been considering introducing Long Term Evolution (LTE) technology. If available, we could design a similar hands-on lab based on LTE. This would provide an interesting option for the students to get hands-on experience with the technology that forms the foundation of the next generation mobile networks.

\section{ACKNOWLEDGMENTS}

This work was supported in part by the NSF GENI project, NSF grants CNS-0916263 and CNS-10-54856, and CIAN NSF ERC under grant EEC-0812072. We would like to express our gratitude to Ivan Seskar from WINLAB at Rutgers University for many useful discussions, and for providing us with critical support while we had been setting up the base station.

\section{REFERENCES}

[1] J. Liebeherr and M. E. Zarki, Mastering Networks: An Internet Lab Manual. Boston, MA, USA: Addison-Wesley Longman Publishing Co., Inc., 2003.

[2] M. Casado and N. McKeown, "The virtual network system," in Proc. ACM SIGCSE'05, Feb. 2005.

[3] N. Sarkar, "Teaching computer networking fundamentals using practical laboratory exercises," IEEE Trans. Educ., vol. 49, no. 2, pp. 285-291, 2006.

[4] J. Prieto-Blazquez, J. Arnedo-Moreno, and J. Herrera-Joancomarti, "An integrated structure for a virtual networking laboratory," IEEE Trans. Ind. Electron., vol. 55, no. 6, pp. 2334-2342, 2008.

[5] S. Abbott-McCune, A. J. Newtson, J. Girard, and B. S. Goda, "Developing a reconfigurable network lab,' in Proc. ACM SIGITE'08, Oct. 2008.

[6] N. Sarkar and T. Craig, "Teaching wireless communication and networking fundamentals using Wi-Fi projects," IEEE Trans. Educ., vol. 49, no. 1, pp. 98-104, 2006.

[7] P. Steenkiste, "The use of a controlled wireless testbed in courses," in Proc. ACM ITiCSE'09, July 2009.

[8] The GENI Project, "Open virtualized WiMAX base station node for GENI wide-area wireless deployments," http:/groups.geni.net/geni/wiki/ WiMAX, 2011, Accessed: Dec. 2012.

[9] Clearwire, http://www.clear.com/, Accessed: Dec. 2012.

[10] OPNET, "Modeler wireless suite," http://www.opnet.com/solutions/ network_rd/modeler_wireless.html, Accessed: Dec. 2012.

[11] P. Garcia-Diaz, S. Salcedo-Sanz, A. Portilla-Figueras, and D. NunezClemente, "GSMSIM: an educational simulation tool for teaching GSMbased mobile communications in laboratory lectures." Int. J. Elec. Eng. Educ., vol. 46, no. 3, pp. 259-279, 2009.

[12] Z. Dawy, A. Husseini, E. Yaacoub, and L. Al-Kanj, "A wireless communications laboratory on cellular network planning," IEEE Trans. Educ., vol. 53, no. 4, pp. 653-661, 2010.

[13] Y. Alqudah and T. Cooklev, "Hands-on open access broadband wireless technology lab," in IEEE EDUCON 2012, Apr. 2012.

[14] K. Etemad and M. Lai, "Overview of WiMAX network architecture and evolution," in WiMAX Technology and Network Evolution, 1st ed. Wiley-IEEE Press, 2010, pp. 147-177.

[15] E. Kohler, R. Morris, B. Chen, J. Jannotti, and M. F. Kaashoek, "The click modular router," ACM Trans. Comput. Syst., vol. 18, no. 3, pp. 263-297, 2000.

[16] S. Seo, J. Janak, and H. Schulzrinne, "Columbia University WiMAX campus deployment and installation," Department of Computer Science, Columbia University, New York, NY, Tech. Rep. CUCS-032-11, June 2011.

[17] K. Etemad and M. Lai, "Overview of mobile WiMAX air interface in release 1.0," in WiMAX Technology and Network Evolution, 1st ed. Wiley-IEEE Press, 2010, pp. 17-51.

[18] WiMAX Forum, "Mobile WiMAX-Part I: A technical overview and performance evaluation," White Paper, Aug. 2006.

[19] WiMax.com Broadband Solutions, Inc., "WiMAX Tutorial: Quality of Service," http://www.wimax.com/wimax-tutorial/quality-of-service, Accessed: Feb. 2012. 


\section{Spring 2012 ELEN E6951 - Wireless \& Mobile Networking II Hands-on Lab \#4 Pre-Lab Questions}

\section{Introduction}

In this experiment we will study the effects of Quality of Service (QoS) class, signal strength, and RF channel contention on the network throughput, packet delay and loss in a 802.16 (WiMAX) mobile wireless network.

\section{Linux Commands}

1. What is ping flooding and how is it different from "normal" pinging?

2. Describe (briefly) the meaning of individual values in: $\mathrm{rtt} \min / \mathrm{avg} / \mathrm{max} / \mathrm{mdev}=80.393 / 80.444 / 80.521 / 0.187$ $\mathrm{ms}$

3. What is the difference between commands ping $-f$ 192.168.0.1 and ping $-\mathrm{s} 1400$-f 192.168.0.1?

\section{PHY layer parameters}

1. What is RSSI? What is CINR? What do these parameters show you?

2. What is the relation between RSSI and CINR to modulation and coding scheme? How does this compare to throughput obtained at WiMAX station?

\section{MAC layer}

See the list of references below for description of the MAC layer in WiMAX and how it can be used to implement various Quality of Service (QoS) classes.

1. How is CSMA (used in WiFi) media access control different from connection-oriented (used in WiMAX) media access control?

2. Can WiMAX MAC guarantee a minimum bandwidth for a mobile station regardless of other traffic on the channel?

3. Which of the two MAC approaches is better for a large number of clients and why?

4. What is the difference between Unsolicited Grant Service (UGS) and Best Effort (BE) Quality of Service classes?

5. Which QoS class would you use for Voice-Over-IP (VoIP) traffic? 


\section{$5 \quad$ Network Architecture}

The following picture illustrates the network architecture we will be using for our experiments. The WiMAX base station is located on the rooftop of Mudd building and is directly connected to a Linux server over a Gigabit Ethernet connection. The antenna is a sector (120 degree) antenna pointed towards the center of the campus. Refer to the section Overview of WiMAX Network Architecture and Evolution in [1] to answer

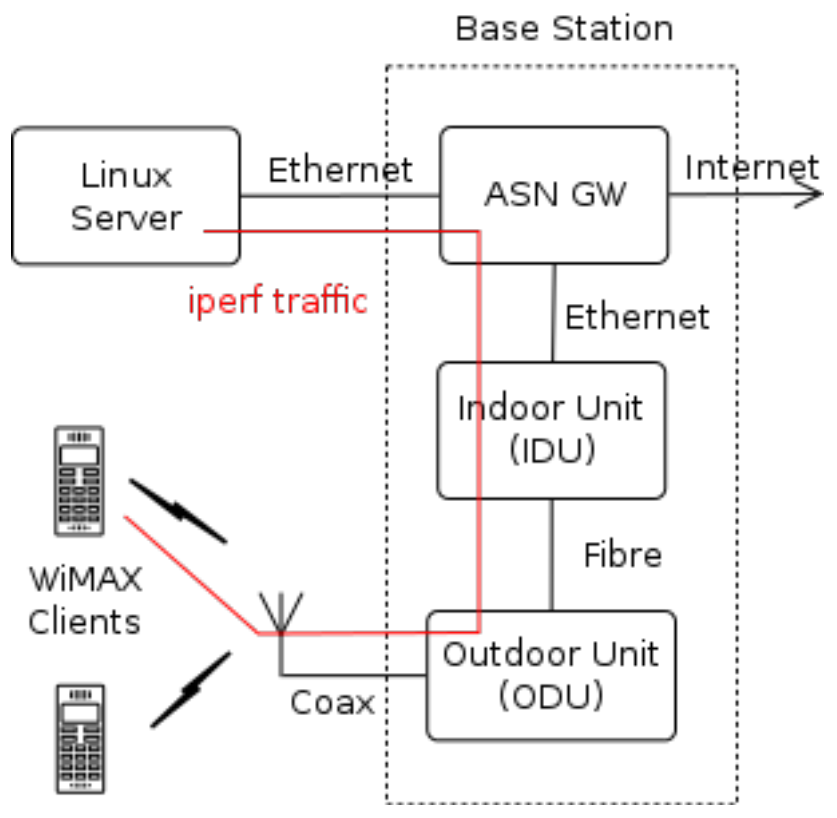

Figure 1: WiMAX Base Station

the following questions:

1. Which of the boxes on the diagram below implements functions related to authorization, mobility management and QoS in a WiMAX network?

2. Which of the links in the diagram below could be labeled as R6 according to the WiMAX network reference model?

\section{References}

[1] Etemad, K., Lai, M. , " WiMAX Technology and Network Evolution" , ch. 6, Wiley-IEEE Press. Available at: http://ieeexplore. ieee.org/xpl/ebooks/bookPdfWithBanner.jsp?fileName= 5732852 .pdf\&bkn=5732785

[2] Etemad, K., Lai, M. , "WiMAX Technology and Network Evolution" , ch. 2, Wiley-IEEE Press. Available at: http://ieeexplore. ieee.org/xpl/ebooks/bookPdfWithBanner.jsp? fileName= 5732857 . pdf\&bkn=5732785

[3] Mobile WiMAX: A Technical Overview. Available at: http://www.wimaxforum.org/technology/ downloads/Mobile_WiMAX_Part1_Overview_and_Performance.pdf

[4] WiMAX QoS http://www.wimax.com/wimax-tutorial/quality-of-service 


\section{ELEN E6951 - Wireless \& Mobile Networking, II \\ Hands-on Lab 4}

Note: please read the entire document carefully before you begin the experiment

\section{Introduction}

This is the fourth hands-on lab of the course. We will experiment "Influence of different traffic classes on throughput, delay and packet loss" in 802.16 wireless network.

(You will be using two laptop machines with WiMAX cards. You can take them from the EE department-13th floor of Mudd.)

\subsection{System Environment}

We are going to use two laptops equipped with WiMAX cards. The bigger Dell laptop has a built-in WiMAX card connected to an antenna above the LCD screen. The smaller Asus laptop has a WiMAX card connected to a USB port. That card has only a small antenna directly on the board.

\begin{tabular}{|l|l|l|}
\hline & \multicolumn{1}{|c|}{ System 1 } & \multicolumn{1}{c|}{ System 2 } \\
\hline Hardware & Dell Laptop & Asus Eee PC \\
\hline OS & Ubuntu 11.04 & Ubuntu 10.10 \\
\hline WiMAX Card & Intel Centrino WiMAX 6250 & Intel Centrino WiMAX 6250 \\
\hline WiMAX Driver & Intel wimaxcu v1.5.1 & Intel wimaxcu v1.5.1 \\
\hline Testing Tool & Iperf 2.0.5 & Iperf 2.0.4 \\
\hline
\end{tabular}

The base station that you will be connecting to is placed on the top of the Mudd building with an antenna facing the center of the campus. Therefore, signal coverage across campus may vary. In general the best coverage can be obtained in outdoor areas around campus, especially at Pupin Plaza and near the Sundial between Shapiro Center and Pupin.

\subsection{Lab Access}

This lab experiment is intended to be done indoor, in the campus area. CVN students can conduct most part of the experiment by remotely accessing ( $\mathrm{SSH}$ ) the two systems.

Locations:

1. CEPSR 714 (Lounge on $7^{\text {th }}$ floor CEPSR), excellent signal, 64-QAM 5/6

2. CEPSR, $4^{\text {th }}$ floor, wooden benches next to the stairs that lead to $120^{\text {th }}$ Street exit 


\subsection{Timeslot Reservation}

Since the entire class is going to share one system setup and the account, we will use Doodle (www.doodle.com) to let you reserve your timeslot to occupy the setup. The reservation is done in first-come-first-serve manner, and you are limited to reserve 1 timeslot (3 hours long) at a time. Lab experiment is done in pairs. Please also refer to the announcement on the CourseWorks.

\section{Hands-on Lab}

Note: Some of the commands you will be running in this lab need to be run in root mode. You can use the sudo command to become root. When asked for a password, type the same password you logged in with.

In this experiment, we will configure two laptops with WiMAX cards to connect to the WiMAX base station at Columbia. The base station is located on the rooftop of Mudd building. One of the laptops has a built-in WiMAX card, while the other laptop has the card connected over USB.

Each of the laptops have been configured with a different Quality of Service class on the base station, thus they will behave differently under load.

Throughout the lab you will need to keep three terminal windows open. Window in which you run client commands such as ping and commands for connecting to/disconnecting from the base station will be referred to as "client terminal window". Window in which you run iperf will be referred to as "iperf terminal window" and window in which you run commands on the base station will be referred to as "base station terminal window".

\subsection{System Setup}

We will do the first part of the experiment on the $4^{\text {th }}$ floor of CEPSR, on wooden benches next to the stairs that lead to $120^{\text {th }}$ street exit from the building. If the battery level on laptops is low you can use plugs on the bottom part of benches. Make sure you run all the steps on both laptop machines.

Step 1: On both laptops open a terminal window (client terminal window) and verify that the WiMAX card is ready and is not connected:

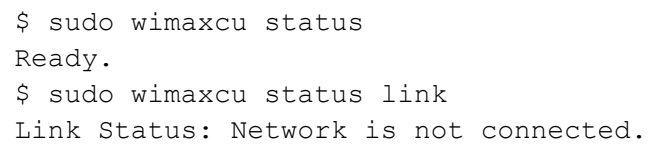

Step 2: Scan for available networks. You should be able to see the GENI 4G network. That's the base station on our campus:

\$ sudo wimaxcu scan

Scanning 0\% Done

Network found. 


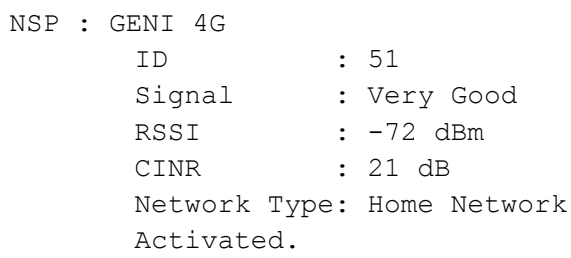

Step 3: On both laptops connect to the WiMAX base station:

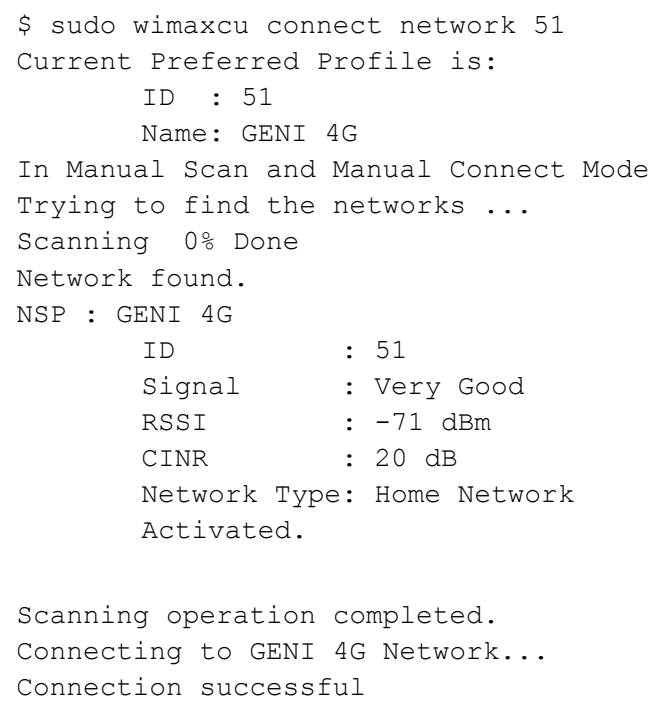

Step 4: On both laptops verify that you're connected to the base station:

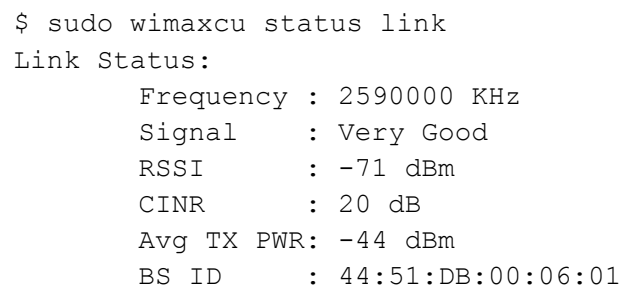

Important: It may happen that you will not be able to connect to the base station if you are in a spot with bad signal coverage. If that is the case then move to a better area and try again.

Step 5: Bring WiMAX interfaces up and configure IP addresses. On the bigger laptop:

\$ sudo ifconfig wmx0 up

\$ sudo ifconfig wmx0 10.0.0.1 netmask 255.255.255.0

\section{On the smaller laptop:}

\$ sudo ifconfig wmx0 up

\$ sudo ifconfig wmx0 10.0.0.2 netmask 255.255.255.0

On both laptops: 
\$ifconfig

Write down MAC addresses of both machines, as you will need them later in the experiment.

Step 6: Open a new terminal window (iperf terminal window) on both laptops and start iperf in server mode:

\$iperf -i $1-u-s$

Keep those terminal windows open. You will need to copy the output of iperf printed there and paste it into the final report.

Step 7: Open a new terminal window (base station terminal window) on the smaller laptop and make sure you can login to the base station:

\$ssh bs

There are two commands that you can run on the base station server to monitor the state of the base station as you run experiments. The command wi_monitor can be used to monitor the state of the wireless channel:

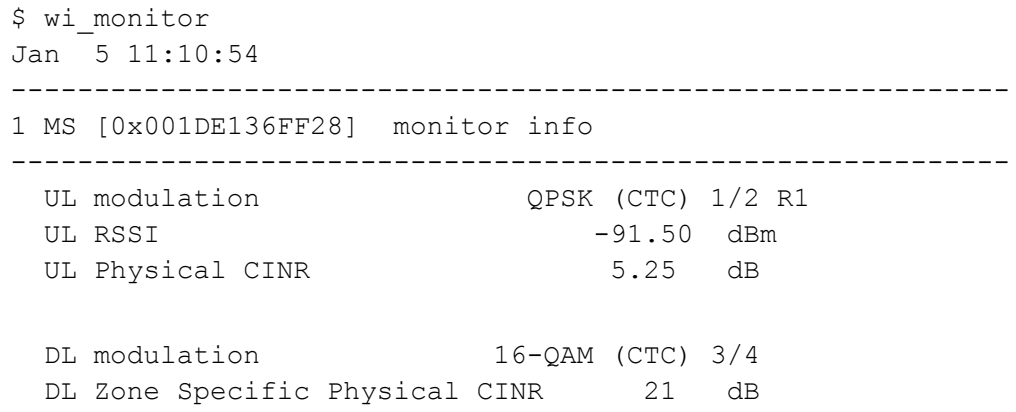

Note that this command shows statistics for all clients connected to the base station. You can find the entry for your client by comparing he MAC address in [] with the MAC address of your WiMAX interface.

The command wi_throughput can be used to obtain information about the current volume of traffic on the channel:

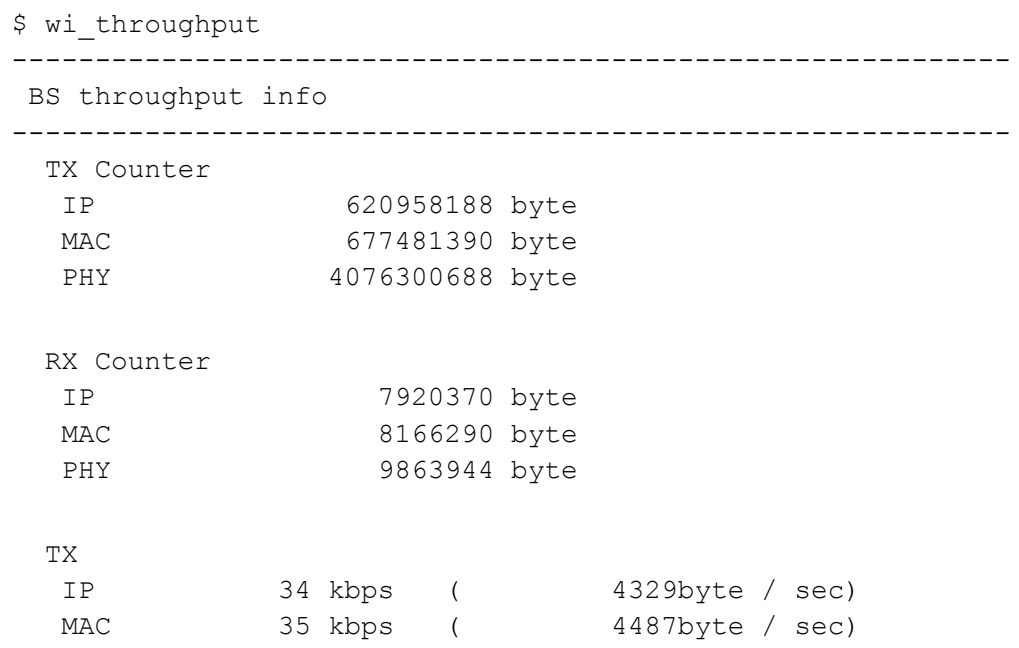




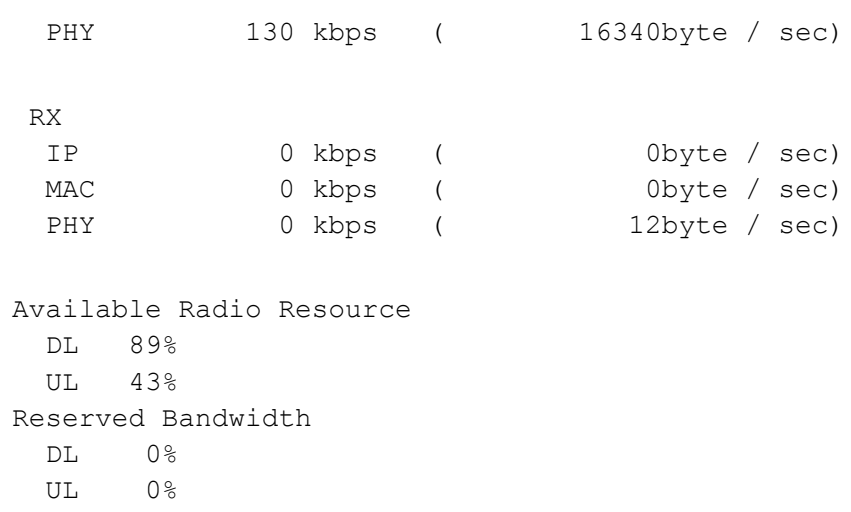

Step 8: On both laptops ping 10.0.0.254 from the client terminal window and observe the delay. Write down the average delay you have observed on each laptop. Type $\mathrm{Ctrl}+\mathrm{C}$ to stop pinging. Include the average round trip time observed on each laptop in your report.

Step 9: On both laptops start ping flooding the base station:

\$ sudo ping -f 10.0.0.254

Every time a packet is sent, a dot is printed; every time a packet is received, a dot is erased. After you quit pinging $(\mathrm{Ctrl}+\mathrm{C})$, you can see the statistics of sent/received packets. What is the percentage of lost packets? Are there any differences in results obtained on both laptops?

Step 10: Now do ping flooding with larger packets:

\$ sudo ping -s 1400 -f 10.0.0.254

What is the percentage of lost packets you see now? Are there any differences in results obtained on both laptops?

\subsection{Maximum Throughput Measurement}

In this part of the experiment we will measure the maximum bandwidth that can be obtained by a single WiMAX client in two different locations. One location has excellent signal reception, allowing the WiMAX base station to pick the most efficient modulation and coding scheme. The other location experiences weaker signal strength, forcing the WiMAX base station to adapt to that situation.

You should stay at the same place where you were taking previous series of steps and run the following set of commands.

Step 1: In the base station terminal window (last one you opened in previous part on the smaller laptop) run wi_monitor command. Find your WiMAX client in the output and note the downlink modulation scheme selected by the base station. Repeat the command several times to ensure 
that the modulation scheme does not change. Copy and paste the output of the command for your client into your final report.

Step 2: On the bigger laptop, go back to the client terminal window (first one you opened) and run "wimaxcu status link" as root:

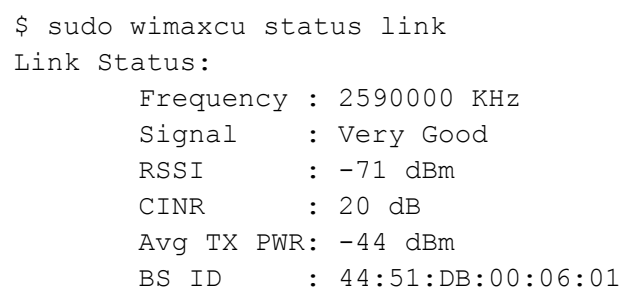

Repeat the command three times and note RSSI and CINR values. Copy and paste the output of the command into your final report.

Step 3: On the smaller laptop, in the base station terminal window (last one opened) run iperf in client mode with the following parameters:

\$ iperf -i 1 -t $10-11440-u-c 10.0 .0 .1-b 4 \mathrm{M}$

Note that it might take some time until what you type in the terminal window shows up on the screen. This is due to low signal level and numerous packets being retransmitted from the base station.

The command above generates a 4Mbit/s stream of data towards the WiMAX client for 10 seconds. You should see iperf server in the terminal window opened on bigger laptop printing traffic statistics every second. If everything goes well then there should be no or minimal packet loss.

Step 4: Repeat the command from the previous step and in each step increase the generated bandwidth by $1 \mathrm{Mbit} / \mathrm{s}$. Keep increasing the bandwidth as long as the packet loss reported by iperf server is below $2 \%$. If you see packet loss over $2 \%$, then the channel is overloaded and you need to step back.

Step 5: Once you have found the maximum bandwidth, repeat the command with -b parameter set to the maximum bandwidth three times to be sure and copy and paste the output of iperf server into your final report.

Step 6: When you are done with the first measurement, take the laptop to the lounge on the $7^{\text {th }}$ floor in CEPSR (room 714), put it on the desk there with the Dell logo on the back of the screen facing the window and repeat steps 1 through 5 from this part of the experiment.

To reconnect to the base station use the set of commands provided in the 2.1 System Setup part of these instructions. 


\subsection{Quality of Service Measurement}

WiMAX supports five Quality of Service (QoS) classes. The goal of this measurement is to explore the effects of two different QoS classes on WiMAX clients. In this experiment we will be using two laptops simultaneously. One of the laptops has been configured to use the Unsolicited Grant Service (UGS) service class, suitable for delay and bandwidth sensitive traffic like VoIP. The other laptop uses the Best Effort (BE) service class commonly used for non-realtime Internet traffic. Your goal is to run a number of ping flooding measurements and tell from the collected data which laptop is UGS and which is BE.

Step 1: On the smaller laptop, run the command wi_monitor in the base station terminal and look at UL RSSI, UL Physical CINR and DL modulation for both WiMAX clients. Move the laptops around on the table, while running wi_monitor repeatedly, so that you get the same DL modulation scheme for both laptops and roughly similar UL CINR and UL RSSI values between the two clients. This is to make sure that both clients experience similar conditions on both uplink and downlink.

Step 2: Run ping -c 5 10.0.0.254 on the smaller laptop and record the command and all its output in your report.

Step 3: Run the same command on the bigger laptop and record its output in your report. Make sure to note which laptop produced which output. Do you see a difference in time values between the two laptops?

Step 4: Run a ping flooding test at both laptops at the same time:

\$ sudo ping -c 500 -f $10 \cdot 0.0 .254$

Include the output of the command from both laptops (especially the $\mathrm{min} / \mathrm{avg} / \mathrm{max} / \mathrm{mdev} \mathrm{rtt}$ numbers) in your report. Repeat this step at least three times to make sure that you get consistent numbers.

After looking at the min/avg/max/mdev numbers produced by the ping command, can you tell which laptop has been configured for the UGS (real-time) service and which is in the BE (best effort) service class?

\subsection{Logout}

Make sure you turn off both laptops and return them to the EE department.

\section{Data Submission and Analysis Report}

Please turn in all the data collected. Label the results properly, so that we can understand the throughput results you obtained in each test scenario (and conditions).

Analyze the data and explain briefly why the result looks that way for each test scenario. In addition, write down your answers to the following questions. 
A. How does the delay you observed when you were pinging the BS compare to the delay you could see when you used WiFi cards (in previous hands-on labs) instead of WiMAX? Can you explain the difference? When you execute ping flooding, do you observe different values packet loss percentage for the two scenarios (steps 9 and 10 of section 2.1)? Why is this the case?

B. What is the difference in the link quality you observed when you performed the experiment at two different locations (CEPSR 714 and $4^{\text {th }}$ floor of CEPSR)? What determines the attainable throughput at different locations? In what case do you get better performance and why?

C. Could you determine which machine was set to work with real-time traffic and which one was set to work as best-effort? What differences in ping delay did you observe? Why are these values different?

\section{References}

Etemad, K., Lai, M. , "WiMAX Technology and Network Evolution”, ch. 6, Wiley-IEEE Press. Available at: http://ieeexplore.ieee.org/xpl/ebooks/bookPdfWithBanner.jsp?fileName $=5732852$.pdf\&bkn $=5732785$

Etemad, K., Lai, M. , "WiMAX Technology and Network Evolution”, ch. 2, Wiley-IEEE Press. Available at: http://ieeexplore.ieee.org/xpl/ebooks/bookPdfWithBanner.jsp?fileName $=5732857$.pdf\&bkn $=5732785$

(end) 